\title{
Inventario de mamíferos medianos y grandes en la reserva los Titíes de San Juan, Montes de María, Bolívar, Colombia
}

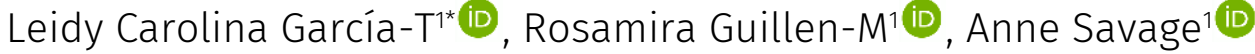 \\ 1 Fundación Proyecto Tití, Calle 77 \# 65 - 37, Local 208, Barranquilla - Colombia. \\ *Correspondencia: Igarcia@proyectotiti.com
}

\section{Resumen}

Reportamos el inventario de mamíferos medianos y grandes para la Reserva Nacional de la Sociedad Civil Los Titíes de San Juan en el departamento de Bolívar. Se registraron un total de ocho ordenes, 18 familias y 22 especies, de los cuales dos se encuentran en peligro de extinción Saguinus oedipus y Ateles fusciceps. Resaltamos la presencia y más reciente registro fotográfico de Galictis vittata. Se destaca la importancia de establecer áreas protegidas que provean conectividad con fragmentos de bosques y protección de especies amenazadas.

Palabras clave: Bosque seco tropical, métodos de muestreo, cámaras trampa

Abstract

We report the inventory of medium and large mammals for the National Reserve of the Civil Society Los Tities de San Juan in the Bolivar department. A total of eight orders were registered, 18 families and 22 species, two of them are in danger of extinction Saguinus oedipus and Ateles fusciceps. We highlight the presence and most recent photographic record of Galictis vittata. We stands out the importance of establishing protected areas that provide connectivity with forest fragments and protection of endangered species.

Key words: Tropical dry forest, sampling methods, camera traps

Los mamíferos juegan un papel ecológico fundamental en el funcionamiento de los ecosistemas, ya sea como polinizadores, dispersores de semillas, y ejerciendo un efecto de control sobre la densidad y abundancia de otras especies (Sinclair 2003). Sin embargo, los mamíferos están cada vez más amenazados debido a la destrucción del hábitat y la cacería (Schipper et al. 2008).

Los bosques secos del Caribe colombiano son uno de los ecosistemas con mayor amenaza en el neotrópico, debido a presiones como deforestación, ganadería, cambios en el uso del suelo, fuego, minería y desarrollo urbano, a tal punto que queda menos de $4 \%$ de su cobertura original (Janzen 1988; Miles et al. 2006; Pizano et al. 2014). En estos bosques, se han registrado 60 especies de mamíferos que corresponden al 12 \% de Colombia (DíazPulido et al. 2014). La riqueza y diversidad para este grupo taxonómico no es muy elevada, 
en comparación a los bosques húmedos, y el conocimiento que se tiene sobre la diversidad de mamíferos en este ecosistema es limitado. Adicionalmente muchos de los estudios que se han realizado no son publicados (Díaz-Pulido et al. 2014). En este contexto, es indispensable contar con información completa y actualizada de la distribución de las especies, para responder preguntas como ¿Cuánto hábitat les queda disponible? ¿Qué tanto han sido impactados por los humanos? Y así, planificar estrategias de conservación, la creación de nuevas áreas protegidas y el establecimiento de programas de educación ambiental y desarrollo sostenible. En este estudio, reportamos el primer inventario de mamíferos medianos y grandes para la Reserva Nacional de la Sociedad Civil Los Titíes de San Juan (RNSC-LTSJ).

El trabajo de campo se realizó en la serranía de San Jacinto, en los Montes de María, Bolívar,

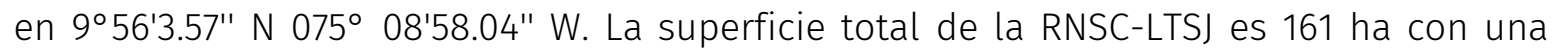
altitud mínima y máxima de 213 y 446 msnm respectivamente, y un promedio anual de temperatura de $27,8^{\circ} \mathrm{C}$. Esta reserva se encuentra dentro de la zona de amortiguación del Santuario de Flora y Fauna Los Colorados (SFFLC) (Figura 1), un parque nacional que protege un fragmento importante de Bosque seco Tropical (BS-T). El inventario se realizó entre oct 2019 - jun 2020. El listado de mamíferos se obtuvo con dos métodos de muestreo: i) Diez cámaras trampa que fueron programadas para tomar videos y fotos (tres fotos consecutivas con intervalos de uno a tres segundos) durante las 24 horas del día, para un esfuerzo de muestreo de 2700 noches/trampa (Figura 1); ii) Observaciones directas a lo largo de dos transectos una vez cada mes (A y B; Figura 1) entre las 06h y las 13h. El transecto A fue de $6 \mathrm{~km}$ y el transecto $B$ fue 3,9 km, para un esfuerzo de muestreo de 89,1 $\mathrm{km}$ recorridos. Finalmente, se calculó la abundancia relativa de los mamíferos reportados en las cámaras trampa (Springer et al. 2012).

Se registraron un total de ocho ordenes, 18 familias y 22 especies de mamíferos grandes y medianos, 17 especies con las cámaras trampa y 10 con las observaciones directas (Figura 1; Tabla 1). Del total de especies documentadas, dos especies de primates están catalogadas como en Peligro Crítico (CR): la Marimonda negra del Chocó Ateles fusciceps (Cortes-Ortíz et al. 2020), y el Tití Cabeciblanco Saguinus oedipus (Rodríguez et al. 2020) un pequeño primate endémico que se distribuye solamente en los bosques tropicales del Caribe colombiano y el Noreste antioqueño, y que se encuentra incluido en el apéndice I de la CITES (CITES c2019). Esta condición de amenaza para las dos especies se debe principalmente a que los primates son muy dependientes de las coberturas vegetales, las cuales se han reducido en los últimos años, a la cacería ilegal para el consumo o la tenencia de especies como mascotas (Rylands et al. 2006).

Dentro de los mamíferos observados en la reserva, resaltamos la presencia y registro fotográfico de Galictis vittata para el departamento de Bolivar, este es un carnívoro poco estudiado en Colombia, con limitada información sobre su distribución, ecología y estado de conservación (Jiménez-Alvarado et al. 2016; Meza-Joya et al. 2018). Las cámaras trampa registraron también al Zorrillo rayado Conepatus semistriatus. Los mustélidos y mefitidos son animales evasivos y poco conspicuos, y a pesar de que representan un elemento fundamental para las comunidades de mamíferos, su conocimiento es muy limitado debido a su naturaleza reservada y las bajas probabilidades de detección, lo cual limita el conocimiento de este grupo en el país, y la evaluación de su estado de conservación (Figura 2; González-Maya et al. 2011; Jiménez-Alvarado et al. 2016). 


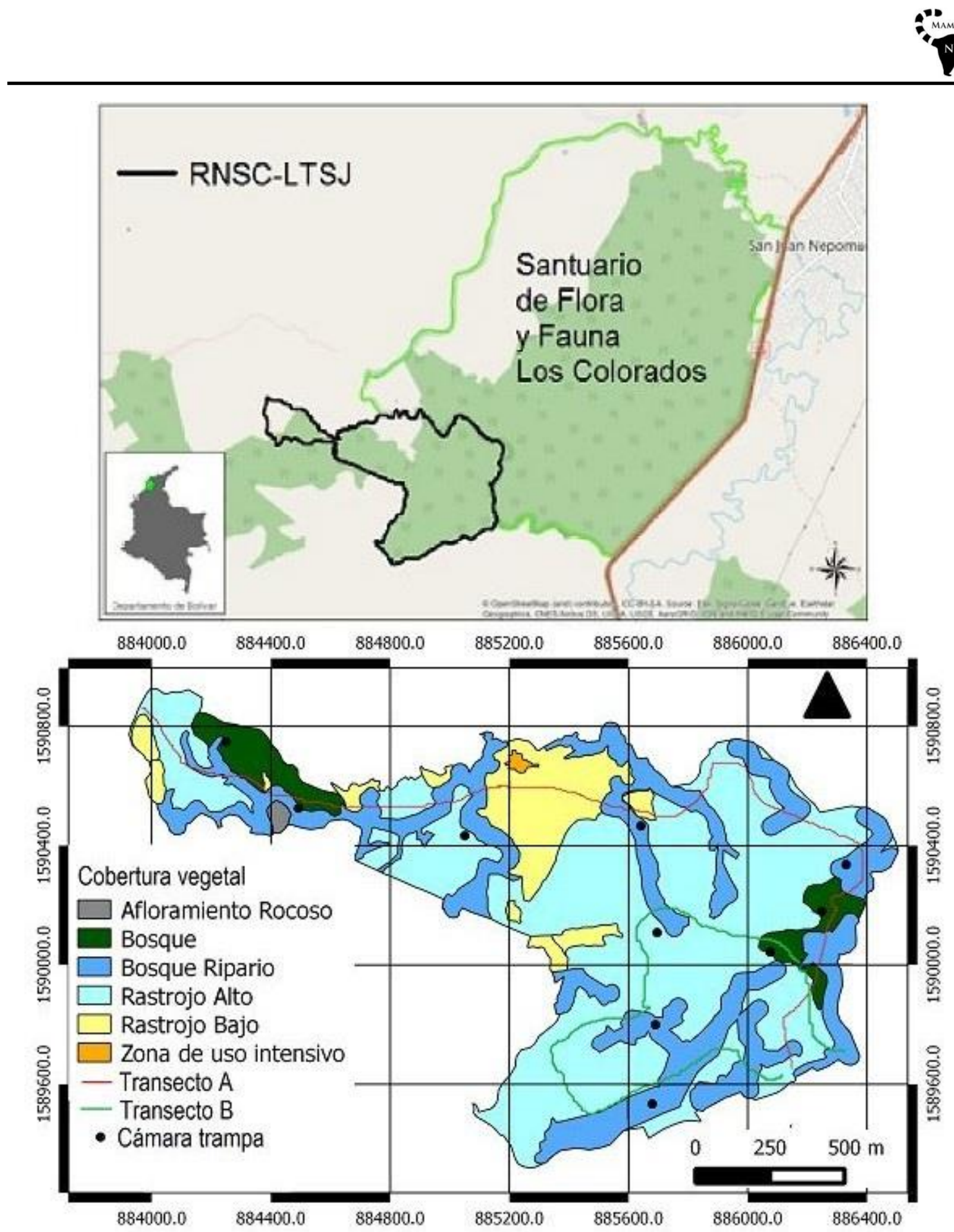

FIGURA 1. Ubicación geográfica de la zona de estudio Reserva Nacional de la Sociedad Civil Los Titíes de San Juan (RNSC-LTSJ) y localización de las trampas cámara y transectos de muestreo. 
TABLA 1. Mamíferos grandes y medianos registrados en la Reserva de la Sociedad Civil Los Titíes de San Juan según su categoría taxonómica, estado de conservación y método de detección (C-T: Cámara-trampa, O-D: Observación directa). Categorías IUCN: DD-Datos Insuficientes; LC- Preocupación menor; CR-Estado Crítico.

\begin{tabular}{|c|c|c|c|c|c|c|c|}
\hline \multirow[t]{2}{*}{ Orden } & \multirow[t]{2}{*}{ Familia } & \multirow[t]{2}{*}{ Especie } & \multirow{2}{*}{$\begin{array}{l}\text { IUCN } \\
2020\end{array}$} & \multirow{2}{*}{$\begin{array}{l}\text { Número } \\
\text { Registros }\end{array}$} & \multirow{2}{*}{$\begin{array}{l}\text { Abundancias } \\
\text { Relativas (\%) }\end{array}$} & \multicolumn{2}{|c|}{ Método } \\
\hline & & & & & & $\mathrm{C}-\mathrm{T}$ & O-D \\
\hline \multirow{2}{*}{ Artiodactyla } & Cervidae & Mazama americana & $\overline{\mathrm{DD}}$ & 90 & 8,21 & $x$ & $\bar{x}$ \\
\hline & Tayassuidae & Pecari tajacu & LC & 142 & 13 & $x$ & - \\
\hline \multirow[t]{7}{*}{ Carnivora } & Felidae & Leopardus pardalis & LC & 44 & 4 & $x$ & $x$ \\
\hline & & Puma yagouaroundi & LC & 4 & 0,36 & $x$ & - \\
\hline & Mephitidae & Conepatus semistriatus & LC & 32 & 3 & $x$ & - \\
\hline & Procyonidae & Procyon cancrivorus & LC & 3 & 0,28 & $x$ & - \\
\hline & Canidae & Cerdocyon thous & LC & 7 & 0,63 & $x$ & - \\
\hline & Mustelidae & Galictis vittata & LC & 2 & 0,18 & $x$ & - \\
\hline & & Eira barbara & LC & 54 & 5 & $x$ & - \\
\hline \multirow[t]{2}{*}{ Cingulata } & Dasypodidae & Dasypus novemcinctus & LC & 72 & 6,6 & $x$ & - \\
\hline & & Cabassous centralis & $\mathrm{DD}$ & 9 & 0,82 & $x$ & - \\
\hline $\begin{array}{l}\text { Didelphimor- } \\
\text { phia }\end{array}$ & Didelphidae & Didelphis marsupialis & LC & 21 & 1,92 & $x$ & \\
\hline \multirow[t]{2}{*}{ Pilosa } & Myrmecopha- & Tamandua mexicana & LC & 18 & 1,64 & $x$ & $x$ \\
\hline & $\begin{array}{l}\text { gidae } \\
\text { Bradipodidae }\end{array}$ & Bradypus variegatus & LC & - & - & - & $x$ \\
\hline \multirow[t]{3}{*}{ Primates } & Atelidae & Alouatta seniculus & LC & - & - & - & $x$ \\
\hline & & Ateles fusciceps & CR & - & - & - & $x$ \\
\hline & Callitrichidae & Saguinus oedipus & $C R$ & - & - & - & $x$ \\
\hline \multirow[t]{4}{*}{ Rodentia } & Cuniculidae & Cuniculus paca & LC & 260 & 23,72 & $x$ & - \\
\hline & Sciuridae & Notosciurus granatensis & LC & 31 & 2,83 & $x$ & $x$ \\
\hline & Erethizontidae & Coendou prehensilis & LC & - & - & - & $x$ \\
\hline & Dasyproctidae & Dasyprocta punctata & LC & 274 & 25 & $x$ & $x$ \\
\hline Lagomorpha & Leporidae & Sylvilagus floridanus & LC & 33 & 3 & $x$ & - \\
\hline
\end{tabular}

Se obtuvieron abundancias relativas altas para Ñeques Dasyprocta punctata, Guartinajas Cuniculus paca, Pecaríes Pecari tajacu y Venados Mazama americana (Tabal 1), lo que sugiere ausencia de depredadores potenciales que tuvieron distribución histórica en la zona como el Jaguar y el Puma, no obstante, estas especies pueden estar siendo consumidas ocasionalmente por felinos como el Jaguarundí Puma yagouaroundi y Ocelote Leopardus pardalis (Emmons 1988; Bianchi et al. 2011). Se registró un total de 22 especies que representa el $36 \%$ de la diversidad de mamíferos para el Bs-T del Caribe colombiano, sin embargo, hay que tener presente que este inventario no tuvo en cuenta los mamíferos pequeños y los voladores, por lo que este numero puede ser significativamente mayor. Si bien, Tinoco-Sotomayor et al. (2016), reportaron para la verada el Camarón y el municipio Santo Domingo en Bolivar 27 especies de mamiferos no voladores, las metodologías usadas difieren en gran medida a las del presente estudio. La RNSC-LTS es un área que provee conectividad del SFFLC con otros fragmentos de bosques aislados que se localizan en áreas aledañas a este parque. Esta reserva pertenece a la Fundación Proyecto Tití, una entidad que busca no solamente la conservación del Tití Cabeciblanco (críticamente amenazado) el cual se ha convertido en una especie sombrilla para la conservación de la diversidad del Bs-T, sino que también busca la protección de su hábitat a partir de proyectos de restauración, generación de corredores biológicos y protección de bosques. 


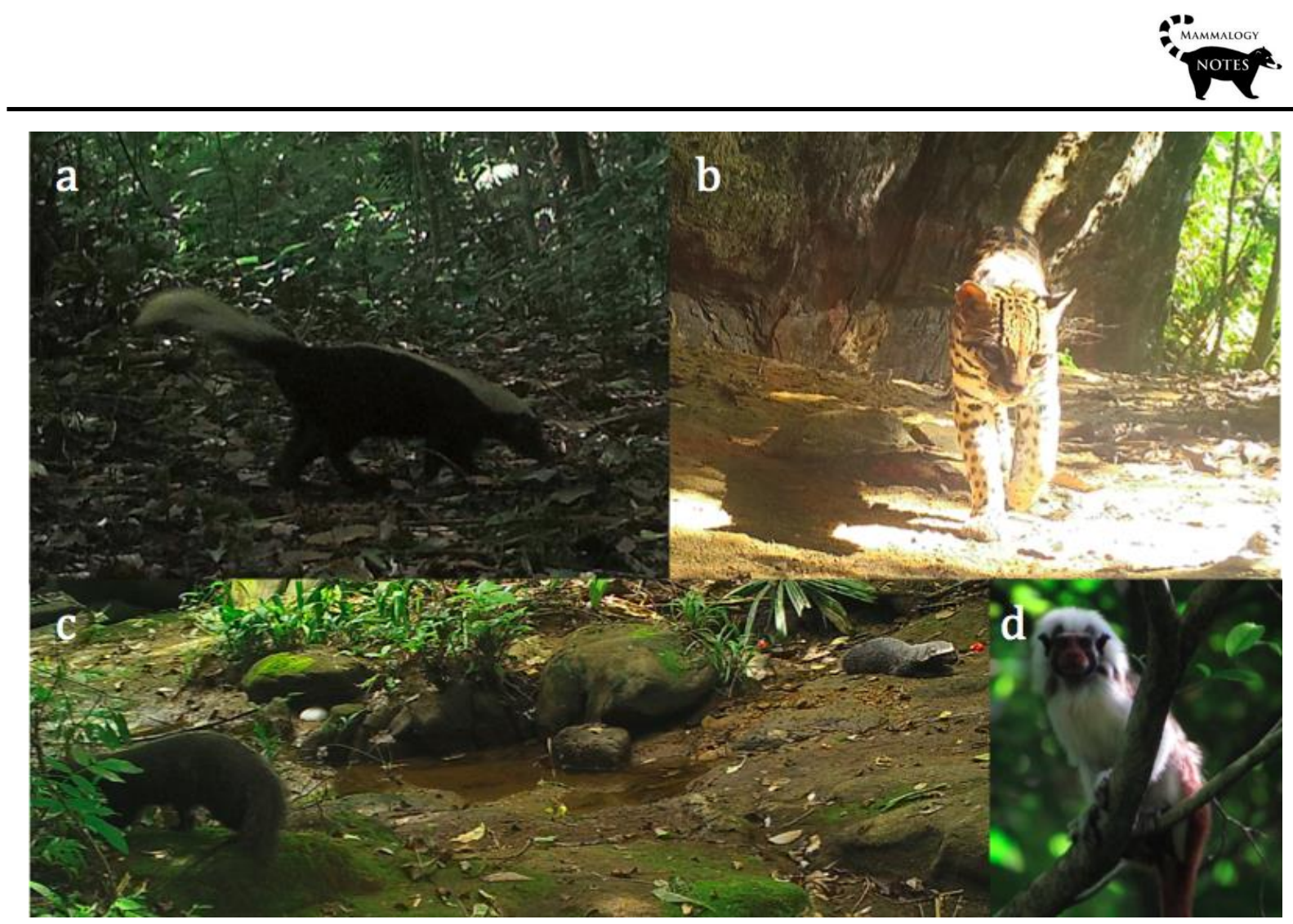

FIGURA 2. Mamíferos medianos y grandes registrados en las cámaras trampa y observaciones directas. a) Conepatus semistriatus, b) Leopardus pardalis, c) Galictis vittata, d) Saguinus oedipus.

\section{AGRADECIMIENTOS}

Los autores agradecen a Rainforest Trust por los fondos destinados en la ejecución de este proyecto, y a los asistentes de campo Germán Emery, Luis Centena, Rodrigo Rodríguez y Aldair Luna.

\section{REFERENCIAS}

CITES. 2019. Convención sobre el comercio internacional de especies amenazadas de fauna y flora silvestres. https://www.cites.org/sites/default/files/esp/app/2019/S-Appendices-2019-1126.pdf. Accessed on 25 July 2020.

Cortes-Ortíz L, Defler TR, Link A, Moscoso P, Méndez-Carvajal P, Shanee S, de la Torre S. 2020. Ateles fusciceps. The IUCN Red List of Threatened Species 2020: https:/ / dx.doi.org/10.2305/ IUCN.UK.2020-2.RLTS.T135446A17929268.en

Bianchi RDC, Rosa AF, Gatti A, Mendes SL. 2011. Diet of margay, Leopardus wiedii, and jaguarundi, Puma yagouaroundi, (Carnivora: Felidae) in Atlantic rainforest, Brazil. Zoologia (Curitiba) 28:127-132. https:// doi.org/10.1590/S1984-46702011000100018

Díaz-Pulido A, Benítez A, Gómez-Ruiz D, Calderón-Acevedo C, Link A, Pardo A, Forero F, De Luna G, Payán E, Solari. 2014. Mamíferos del bosque seco, una mirada al Caribe colombiano. En: Pizano C, García H, editores. El bosque seco tropical en Colombia. Bogotá, Colombia: Instituto de Investigación de Recursos Biológicos Alexander von Humboldt (IAvH). p. 128165.

Emmons L. (1988). A field study of ocelots (Felis pardalis) in Perú. Revue d'écologie 43:133-157. http://hdl.handle.net/2042/55319 
González-Maya JF, Cepeda AA, Belant JL, Zárrate-Charry DA, Balaguera-Reina SA, Rodríguez-Bolaños A. 2011. Research priorities for the small carnivores of Colombia. Small Carnivore Conservation 44:7-13.

Janzen DH. 1988. Management of habitat fragments in a tropical dry forest: growth. Annals of the Missouri botanical garden 75:105-116. https:// doi.org/10.2307/2399468

Jiménez-Alvarado JS, Arias-Ocampo A, Pineda-Guerrero A, Zárrate-Charry DA, Vela-Vargas IM, Chacón-Pacheco J, González-Maya JF. 2016. Análisis de la distribución del grisón (Galictis vittata) (Carnivora: Mustelidae) en el Caribe colombiano. Therya 7(1):179-186. https:// doi.org/10.12933/therya-16-303

Meza-Joya FL, Ramos E, Cediel F, Martínez-Arias V, Colmenares J, Cardona D. 2018. Predicted distributions of two poorly known small carnivores in Colombia: the greater grison and striped hog-nosed skunk. Mastozoología neotropical 25: 89-105. https:/ / doi.org/10.31687/ saremMN.18.25.1.0.09

Miles L, Newton AC, Defries RS, Ravilious C, May I, Blyth S, Kapos V, Gordon JE. 2006. A global overview of the conservation status of tropical dry forests. Journal of Biogeography 33:491-505. https:/ / doi.org/10.1111/j.1365-2699.2005.01424.x

Pizano C, García H. 2014. El Bosque Seco Tropical en Colombia. Instituto de Investigación de Recursos Biológicos Alexander Von Humboldt (IAvH), Bogotá, Colombia.

Rodríguez V, Defler TR, Guzman-Caro D, Link A, Mittermeier RA, Palacios E, Stevenson PR. 2020. Saguinus oedipus. The IUCN Red List of Threatened Species 2020: https:/ / dx.doi.org/10.2305/IUCN.UK.2020-2.RLTS.T19823A115573819.en.

Rylands AB, Groves CP, Mittermeier RA, Cortés-Ortiz L, Hines JJ. 2006. Taxonomy and Distributions of Mesoamerican Primates. In: Estrada A, Garber P, Pavelka M, Luecke L, editors. New perspectives in the study of Mesoamerican primates. New York: Springer 29-79. https:// doi.org/10.1007/0-387-25872-8_3

Schipper J, Chanson JS, Chiozza F, Cox NA, Hoffmann M, Katariya V, Baillie J. 2008. The status of the world's land and marine mammals: diversity, threat, and knowledge. Science 322: 225-230. https:// doi.org/10.1126/science.1165115

Sinclair A. 2003. The role of mammals as ecosystem landscapers. Alces. 39:161-176.

Springer MT, Carver AD, Nielsen CK, Correa NJ, Ashmore JR, Ashmore JR, Lee JG. 2012. Relative abundance of mammalian species in a central Panamanian rainforest. Latin American Journal of Conservation 3:19-26.

Terborgh J, Lopez L, Nuñez P, Rao M, Shahabuddin G, Orihuela G, Balbas L. 2001. Ecological meltdown in predator-free forest fragments. Science 29:1923-1926. https:// doi.org/10.1126/science.1064397

Tinoco-Sotomayor AN, Ramos-Guerra HD, Vides-Avilez HA, Rodríguez-Alarcón DC, González-Maya JF, Gómez-Estrada H. 2016. Inventario preliminar y uso de mamíferos silvestres no voladores en la vereda Camarón, Montes de María (Bolivar-Colombia). Mammalogy Notes 3(1-2):32-36. https://doi.org/10.47603/manovol3n1.32-36

Editor: Diego J. Lizcano Recibido: 2020-05-03

Revisado: 2020-05-15

Aceptado: 2020-08-05

Publicado: 2020-12-24 